\title{
Plasmon Enhanced Internal Quantum EFFICIENCY OF CdSe/ZnS QuANTUM DotS
}

\author{
Sioma Debela, Teshome Senbeta and Belayneh Mesfin \\ Department of Physics, Addis Ababa University, Addis Ababa, Ethiopia
}

\begin{abstract}
The effects of surface plasmon oscillations on the radiative recombination rate and internal quantum efficiency of silver coated CdSe/ZnS quantum dots imbedded in quartz matrix is studied theoretically and numerically. The analysis is carried out by introducing the local field enhancement factor for the description of the dielectric function of the quantum dots together with the modified Drude model for the metal-coat that takes into account the effects of interband transitions and the size dependent damping parameter. It is found out that the internal quantum efficiency of photoluminescence emission of the metalcoated CdSe/ZnS quantum dots is enhanced by about 5-folds compared with the quantum dots without metal-coat. Moreover, increasing the metal fraction of the coated CdSe/ZnS quantum dots from 0.80 to 0.92 results in a significant increase of the intensity that is accompanied by a blue-shift of the quantum efficiency curves. The results obtained can be utilized in the design and fabrication of optoelectronic devices that require high intensity of photoluminescence emission.
\end{abstract}

\section{KEYWORDS}

Photoluminescence, Plasmon, Internal quantum efficiency, Recombination rate

\section{INTRODUCTION}

Among the various luminescent materials, semiconductor quantum dot (QD) nanocrystals have attracted a great deal of attention because of their size-tunable photoluminescence (PL), high PL quantum yields and high photostability [1-3]. In particular, CdSe-based QD nanocrystals are very promising materials as optoelectronic devices such as light emitting sources and a biological fluorescent devices [4,5]. Despite CdSe-based QDs possesses a number of advantageous characteristics over the traditional fluorescent devices, their light emission efficiencies are relatively low $[3,5]$. The most important requirement for a competitive nanostructured light emitting device is high quantum efficiency of light emission. Among the parameters that describes the light emission efficiency is the internal quantum efficiency $\left(\eta_{\text {int }}\right)$, which is defined as the ratio of radiative recombination rate to the total radiative and non-radiative recombination rates. Hence, it is obvious that an increase in $\eta_{\text {int }}$ can be achieved either by decreasing the nonradiative recombination rate $\left(\Gamma_{n r}\right)$ or increasing the radiative recombination rate $\left(\Gamma_{r r}\right)$.

In CdSe-based QDs, a reduction in $\Gamma_{n r}$ is achieved by capping the QDs with wide gapped semiconductors such as $\mathrm{ZnS}$ and CdS. The carriers generated by the incident radiation are strongly localized in the CdSe core in the semiconducting shell. This prevents the carriers from being captured in the nonradiative recombination sites [2,6,7]. On the other hand, an increase in $\Gamma_{r r}$ is achieved via surface plasmon (SP) coupling by coating the CdSe/ZnS structure with noble metals such as silver and gold. In metal coated QDs, the electron-hole pairs generated in the QDs 
couple to electron vibrations at the metal surface when the frequencies of electron-hole pairs in the QDs and of the metal SP are similar. Such SP enhancement mechanisms of the $\eta_{\text {int }}$ of QDs are reported in many experiments [8-10].

In this paper, we study the effects of Surface Plasmon on $\Gamma_{r r}$ and $\eta_{\text {int }}$ of a system of silver coated CdSe/ZnS QDs imbedded in quartz matrix. The analysis is carried out by introducing (i) the modified Drude model for the dielectric function of plasmonic metals that takes into account (a) the size dependent damping parameter and (b) the effect of interband transitions; and (ii) the local electric field enhancement factor for the dielectric function of the semiconductor QDs. The system shown in Fig. 1 illustrates the different layers of the silver coated CdSe/ZnS QD. The wide gapped $\mathrm{ZnS}$ shell passivates the nonradiative recombination sites and the metal coat enables SP coupling with the energy gap of the QDs.

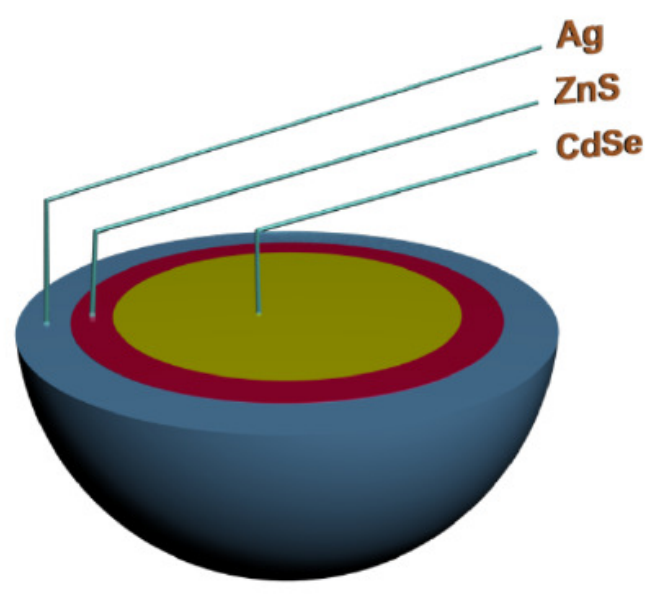

Figure 1. Schematic diagram of the silver coated quantum dot hemisphere. The central portion represents the QD, the middle layer is the barrier, and the outer shell is silver.

The paper is tailored as follows. In Section 2, we consider the enhanced $\Gamma_{r r}$ in silver coated nano-emitters and the competitive nonradiative recombination rate, $\Gamma_{n r}$. The theoretical and numerical analysis of the surface plasmon enhanced $\eta_{\text {int }}$ of silver covered nano-emitters is carried out in Section 3. The results obtained in the paper are summarized in Section 4.

\section{RADIATIVE AND NON-RADIATIVE RECOMBINATION RATES}

Consider a quantum dot that is irradiated by an incident electromagnetic waves (EMWs). The rate of radiative quantum transition $\Gamma_{r r}$ from an excited state $|c\rangle$ to the ground state $|v\rangle$ is described by the Fermi's golden rule [11] $\Gamma_{r r}=(2 \pi / \hbar)\left[\left|\left\langle v\left|\hat{H}_{i}\right| c\right\rangle\right|^{2} \delta\left(E_{c}-E_{v}-\hbar \omega\right)\right]$, where $\hat{H}_{i}$ is the operator of the interaction Hamiltonian, $\hbar \omega$ is the emitted photon energy, $E_{c}$ and $E_{v}$ are the energies of the lowest unoccupied molecular orbital (LUMO), and the highest occupied molecular orbital (HOMO), respectively. 
If the QDs size is much smaller than the wavelength of the incident light, then the operator of the interaction Hamiltonian may be written as $\hat{H}_{i}=-\hat{\mathbf{d}} \cdot \mathbf{E}(\omega) \exp (-i \omega t)$, where $\hat{\mathbf{d}}$ is the operator of electric dipole moment and $\mathbf{E}(\omega)$ is the amplitude of the electric field of the incident EMW of frequency $\omega$.

For CdSe QDs, the supra-linear dependency of the radiative recombination rate on the emission frequency $\omega$ obtained using the Fermi's golden rule [12] is given by

$$
\Gamma_{r r}=\frac{C \omega}{1+\Theta r_{d}^{3}}
$$

where $C$ is a constant, $\Theta=\left(2 m * k_{B} T / \hbar^{2}\right)^{3 / 2} /(6 \sqrt{\pi}), \hbar$ is the Planck's constant, $k_{B}$ is the Boltzmann's constant, $T$ is the temperature, $m^{*}$ is the hole effective mass, and $r_{d}$ is the radius of a QD.

For metal coated QDs the local field, $E_{L}$, is given by $E_{L}(\omega)=F(\omega) E(\omega)$, where $F(\omega)$ is the enhancement factor. In particular, for spherical metal coated QDs in a dielectric matrix, the enhancement factor takes the form [13]

$$
F(\omega)=\frac{9 \varepsilon_{h} \varepsilon_{m}}{2 p \Delta} .
$$

Here $p=1-\left(r_{d} / r_{m}\right)^{3}, \Delta=\varepsilon_{m}^{2}+q \varepsilon_{m}+\varepsilon_{d} \varepsilon_{h}$, and $q=[3 /(2 p)-1] \varepsilon_{d}+(3 / p-1) \varepsilon_{h}$, where $p$ is the metal fraction, $r_{d}$ is the radius of the $\mathrm{QD}, r_{m}$ is the radius of the $\mathrm{QD} / \mathrm{ZnS} /$ metal-coat structure, and $\varepsilon_{d}, \varepsilon_{m}$, and $\varepsilon_{h}$ are the dielectric functions of the QD, metal coat, and host matrix; respectively. For the sake of simplicity, we chose $\varepsilon_{d}$ and $\varepsilon_{h}$ to be frequency independent constants.

In addition, the frequency dependent dielectric function of plasmonic metals based on a critical point analysis of the actual metal band structure was discussed by D. Rioux, et al [14]. Accordingly, we choose $\varepsilon_{m}$ to have the modified Drude form given by

$$
\varepsilon_{m}=\varepsilon_{\infty}-\frac{\omega_{p}^{2}}{\omega(\omega+i \gamma)}+\varepsilon_{c p 1}\left(\omega, \omega_{01}, \omega_{g 1}, \gamma_{1}, A_{1}\right)+\varepsilon_{c p 2}\left(\omega, \omega_{02}, \gamma_{2}, A_{2}\right)
$$

Here, $\varepsilon_{\infty}$ is some real constant, $\omega_{p}$ is the plasma frequency of the metal, $\gamma$ is the decay constant of plasma vibrations, $\varepsilon_{c p 1}$, and $\varepsilon_{c p 2}$ are the interband contributions to the dielectric function of plasmonic metals at the $\mathrm{M}_{1}$ and $\mathrm{M}_{2}$ critical points of the metal band structure, respectively (with $\omega_{01}, \omega_{g 1}, \gamma_{1}, A_{1}$ are the $\mathrm{X}$ symmetry point threshold transition frequency, gap frequency, plasma broadening factor, and amplitude parameter, respectively, and $\omega_{02}, \gamma_{2}, A_{2}$ are the $\mathrm{L}$ symmetry point threshold transition frequency, plasma broadening factor, and amplitude parameter; respectively). Typical values of these parameters for silver are shown in Table 1. Moreover, for small metal particles of size comparable to or smaller than the mean free path of conduction electrons, $\gamma$ is a function of the particle radius, $a[15,16]$. That is,

$$
\gamma=\gamma_{b u l k}+A \frac{v_{F}}{a},
$$


International Journal of Recent advances in Physics (IJRAP) Vol.5, No.2, May 2016

where $\gamma_{\text {bulk }}$ is the bulk decay constant of plasma vibrations, $v_{F}$ is the velocity of the electrons at the Fermi surface, and $A$ is a parameter which depends on the details of the scattering process $[17,18]$.

Table 1. Typical values of the parameters that accounts the interband contribution to the dielectric function of silver nanostructure. [14]

\begin{tabular}{|c|c|c|c|c|c|c|}
\hline$\omega_{g 1}(\mathrm{eV})$ & $\omega_{01}(\mathrm{eV})$ & $\omega_{02}(\mathrm{eV})$ & $\gamma_{1}(\mathrm{eV})$ & $\gamma_{2}(\mathrm{eV})$ & $A_{1}$ & $A_{2}$ \\
\hline 4.0575 & 4.1655 & 0.0224 & 0.0177 & 0.1882 & 51.2170 & 30.770 \\
\hline
\end{tabular}

Taking into account of the enhanced local electric field described by Eq. (2), the rate of radiative quantum transition $\Gamma_{r r}$ in the metal coated QDs takes the following form:

$$
\tilde{\Gamma}_{r r}=\frac{C \omega}{1+\Theta r_{d}^{3}}|F(\omega)|^{2}
$$

where the square of the enhancement factor $|F(\omega)|^{2}$ that can be obtained from Eq. (2) is given by

$$
|F(\omega)|^{2}=\frac{81 \varepsilon_{h}^{2}}{4 p^{2}} \frac{\varepsilon_{m}^{\prime 2}+\varepsilon_{m}^{\prime \prime 2}}{\left(\varepsilon_{m}^{\prime 2}+q \varepsilon_{m}^{\prime}+\varepsilon_{d} \varepsilon_{h}-\varepsilon_{m}^{\prime \prime 2}\right)^{2}+\varepsilon_{m}^{\prime \prime 2}\left(q+2 \varepsilon_{m}^{\prime}\right)^{2}}
$$

Here $\mathcal{E}^{\prime}{ }_{m}$ and $\mathcal{E}^{\prime \prime}{ }_{m}$ are the real and imaginary parts of $\varepsilon_{m}$ defined by (3). From Eq. (5), it is obvious that for $|F(\omega)|^{2} \gg>1$, the rate of radiative quantum transitions in metal coated QDs is considerably enhanced compared to the un-coated ones.

For a system where the space between the QDs in the system is sufficiently large, all other interactions may be neglected so that radiative recombination occurs only between states within individual QDs. Consequently, applying the WKB approximation in the high potential barrier limit [19], the non-radiative recombination rate $\Gamma_{n r}$ is found to be

$$
\Gamma_{n r}=\Gamma_{0} e^{\alpha E},
$$

where $\Gamma_{0}=k \exp \left(-2 \alpha V_{0}\right), \alpha=\ell \sqrt{2 m^{*} /\left(V_{0} \hbar^{2}\right)}, E$ is the PL energy, $k$ is the proportionality constant, $V_{0}$ is the potential height of the barrier, $\ell$ is the thickness of the barrier, and $m *$ is the effective mass of electrons or holes in the barrier.

Table 2. Typical values for the parameters of the QD/metal structure. [20,21]

\begin{tabular}{|c|c|c|c|c|c|c|c|}
\hline$V_{0}(\mathrm{eV})$ & $\omega_{p}(\mathrm{eV})$ & $\gamma_{\text {bulk }}(\mathrm{eV})$ & $\varepsilon_{h}$ & $\varepsilon_{d}$ & $\varepsilon_{\infty}$ & $v_{F}(\mathrm{~m} / \mathrm{s})$ & $A$ \\
\hline 1.30 & 8.5546 & 0.0224 & 3.80 & 6.10 & 1.74 & $1.4 \times 10^{6}$ & 1 \\
\hline
\end{tabular}




\section{Plasmon ENHANCED INTERNAL QUANTUM EFFICIENCY}

The frequency dependent $\eta_{\text {int }}$ is given by

$$
\eta_{\text {int }}(\omega)=\frac{\Gamma_{r r}(\omega)}{\Gamma_{r r}(\omega)+\Gamma_{n r}(\omega)} .
$$

Substituting Eqs. (1) and (7) into Eq. (8), we obtain $\eta_{\text {int }}$ of the QDs without metal coat

$$
\eta_{\text {int }}(\omega)=\frac{\omega}{\Omega\left(1+\Theta r_{d}^{3}\right) e^{\beta \omega}+\omega},
$$

where $\beta=\alpha \hbar$ and is the ratio of $\Gamma_{0}$ to $\Gamma_{r r}$.

Similarly, substituting Eqs. (5) and (7) into Eq. (8) and manipulating using Eq. (9), we obtain the internal quantum efficiency of the metal coated QDs, $\tilde{\eta}_{\text {int }}(\omega)$, in terms of $\eta_{\text {int }}(\omega)$ to be

$$
\tilde{\eta}_{\text {int }}(\omega)=\frac{\eta_{\text {int }}(\omega)|F(\omega)|^{2}}{\eta_{\text {int }}(\omega)\left(|F(\omega)|^{2}-1\right)+1} .
$$

Equation (10) shows that for $|F(\omega)|^{2}>>1, \tilde{\eta}_{\text {int }}(\omega)$ of metal coated QDs is considerably enhanced compared with the QDs without metal coat. It is worth noting that, to the best of our knowledge, Eq. (10) is a new equation which introduces the local field enhancement factor for $\mathrm{CdSe} / \mathrm{ZnS}$ QDs together with the modified Drude model for the metal coat that takes into account the effect of interband transitions and the size dependent damping parameter. Below, we analyze numerically the $\eta_{\text {int }}$ of the CdSe/ZnS QDs with and without metal coats as a function of the emission frequency, for typical parameters of the nanostructure.

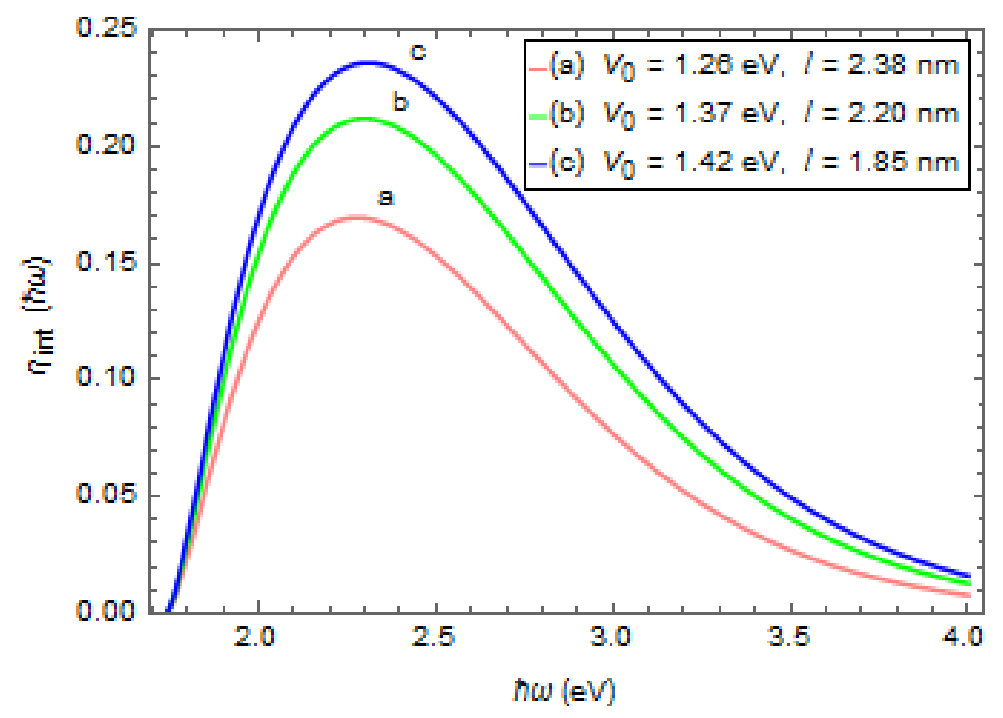

Figure 2. The $\eta_{\text {int }}$ of the uncoated CdSe/ZnS QDs versus the emission energy ( $\hbar \omega$ ) for three values of the barrier height $V_{0}$ and barrier thickness $\ell, \Omega=0.021, m^{*}=0.13 m_{e}$, and $T=300 \mathrm{~K}$.

The internal quantum efficiency of the CdSe/ZnS QDs without metal coat versus the emission energy is shown in Fig.2, for three different values of barrier height $V_{0}$ and barrier thickness $\ell$. It is observed that as the barrier as the barrier height is increased with a corresponding decrease in 
the barrier thickness, $\eta_{\text {int }}(\omega)$ is increased. Moreover, the peak of $\eta_{\text {int }}(\omega)$ curves is accompanied by slight blue shift with an increase in the barrier height. The blue shift is mainly associated with increased quantum confinement energy as a result of a decrease in the size of the QDs.

Figure 3 shows the plasmon enhanced $\tilde{\eta}_{\text {int }}$ versus the emission energy for silver coated $\mathrm{CdSe} / \mathrm{ZnS}$ QDs imbedded in quartz matrix. A significant enhancement of $\tilde{\eta}_{\text {int }}$ of the silver coated $\mathrm{CdSe} / \mathrm{ZnS}$ QDs is observed compared with the QDs without a metal coat. For instance, comparison of curves c in Figs. 2 and 3 show that $\tilde{\eta}_{\text {int }}$ of the silver coated CdSe/ZnS QDs is about 5-folds greater than that of the QDs without a metal coat. This significant enhancement is mainly attributed to strong coupling of surface plasmons oscillations with the frequency of the radiative transitions of the generated electron-hole pairs.

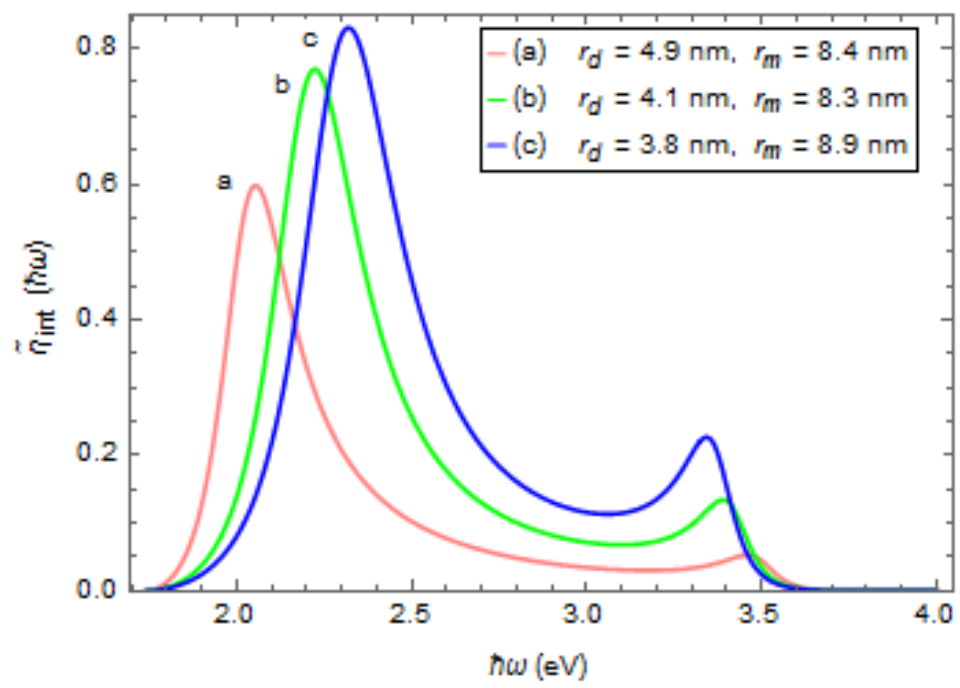

Figure 3. Plasmon enhanced $\tilde{\eta}_{\text {int }}$ versus the emission energy for silver coated CdSe/ZnS QDs imbedded in quartz matrix for three resonant values of $r_{d}$ and $r_{m}$; using the parameters of Fig.2 and those shown in Tables 1 and 2.

Moreover, unlike that of the QDs without metal coat, the $\tilde{\eta}_{\text {int }}$ curves of the metal coated QDs have two sets of peaks, i.e., the first set of peaks for energies below $2.4 \mathrm{eV}$ and the second set of peaks for energies above $3.3 \mathrm{eV}$; as shown in Fig.3. The first peaks are due to the core CdSe dots and the second peaks are due to the interaction between the $\mathrm{ZnS}$ shell and the host matrix. The appearance of the second set of peaks is because of the silver coat which resulted in significant enhancement of the radiative recombination in the dots. It is observed that the first set of peaks are blue shifted with a decrease of the size of the QDs. When the metal fraction $p$ increases, the size of QD decreases, which in turn increases the energy gap of the QD. This increase in energy gap of the QDs approach the surface plasmon energy of the coated metals; however, since the surface plasmon energy is still higher than the energy gap of the QDs, it results to an increased blue shift in the $\tilde{\eta}_{\text {int }}$ curves. This phenomena is illustrated in Fig.3 which shows that an increase in the metal fraction from 0.80 to 0.92 results to a clear blue shift in the $\tilde{\eta}_{\text {int }}$ curves; the blue shift being more apparent with an increased $\widetilde{\Gamma}_{r r}$ associated with stronger coupling between the energy gap of the emitter and the surface plasmon energy of silver. On the other hand, the second set of 
International Journal of Recent advances in Physics (IJRAP) Vol.5, No.2, May 2016

peaks are red shifted with an increase in the metal fraction, $p$, with the maxima of the second set of peaks becoming more pronounced with increasing metal fraction.

\section{Conclusions}

In this paper we studied the plasmon enhanced $\eta_{\text {int }}$ of PL emission of CdSe/ZnS QDs with and without the metal coat. It is shown that when QDs are coated with silver, the local field enhances remarkably, resulting to appreciably increase the $\Gamma_{r r}$ and PL intensity. This enhancement is mainly attributed to the strong coupling of the surface plasmons oscillations of the silver with the energy gap of the CdSe/ZnS QDs.

It is found out that the $\tilde{\eta}_{\text {int }}(\omega)$ and the corresponding PL emission is increased significantly by increasing the metal fraction $p$ of the QDs. In particular, for $p=0.92$, the $\tilde{\eta}_{\text {int }}(\omega)$ increases by about 5-folds with respect to the QDs without a metal coat. Also, it is shown that the $\eta_{\text {int }}$ of the plasmon coupled QDs increases significantly possessing two sets of peaks compared with the QDs without metal coat.

It may be noted that with appropriate choice of the metal fraction, size of QDs, the potential height of the barrier and frequency of the incident light, one can achieve optimum PL intensity from metal coated QDs. We claim that the results obtained in this paper can potentially be employed in the design and fabrication of noble metal coated QDs for application in optoelectronic devices that require high quantum efficiency of PL emission.

\section{ACKNOWLEDGEMENTS}

This work is dedicated to the late Professor V. N. Mal'nev who departed suddenly on January 22, 2015. We greatly acknowledge his invaluable contributions right from problem setting to almost its conclusion. Let his soul rest in peace. 
International Journal of Recent advances in Physics (IJRAP) Vol.5, No.2, May 2016

\section{REFERENCES}

[1] Demir, H.V.; Nizamoglu, S.; Mutlugun, E.; Ozel, T.; Sampra, S.; Gaponik, N. and Eychmuller, A. (2008) Nanotechnology, 19, 335203.

[2] Grabolle, M.; Ziegler, J.; Merkulov. A.; Nann, T. and U. Resch-Genger (2008) Ann. N.Y. Acad. Sci., 1130, 235241 and reference therein.

[3] Cordero, S.R.; Carson, P.J.; Estabrook, R.A.; Strouse, G.F. and Buratto, S.K. (2002) J. Phys. Chem. B, 104, 51 and reference therein.

[4] Pilla, V.; Alves, L.P.; Munin, E.; Pacheco M.T.T. (2007) Opt. Commun., 280, 225229 and reference therein.

[5] Okamoto, K.; Vyawahare, S. and Scherer, A. (2006) J. Opt. Soc. Am. B, 23, 8.

[6] Dabbousi, B.O.; J. Rodriguez-Viejo; Mikulec, F.V.; Heine, J.R.; Mattoussi, H., Ober, R.; Jensen, K.F. and Bawendi, M.G. (1997) J. Phys. Chem. B, 101, 46.

[7] Mathew, S.; Bhardwaj, B.S.; Saran, A.D. and Radhakrishnan, P. (2015) Opt. Mater., 39, 4651.

[8] Kulakovich, O.; Strekal, N.; Yaroshevich, A.; Maskevich, S., Gaponenko, S.; Nabiev, I.; Woggon, U. and Artemyev, M. (2002) Nano Lett., 2, 1449-1452.

[9] Song, J.H.; Atay, T.; Shi, S.; Urabe, H. and Nurmikko, A.V. (2005) Nano Lett., 5, 1227-1561.

[10] Gryczynski, I.; Malicka, J.; Jiang, W.; Fischer, F.; Chan, W.C.W.; Gryczynski, Z.; Grudzinski, W. and Lakowicz, J.R. (2005) J. Phys. Chem. B, 109, 1088-1093.

[11] Capasso, F., (2002); Academic press, San Diego.

[12] Van Driel, A.F.; Allan, G.; Delerue, C.; Lodahl, P.; Vos, W.L. and Vanmaekelbergh, D. (2005) Phys. Rev. Lett., 95, 236804.

[13] Shewamare, S; Mal'nev, V.N. (2012) Phys.B:Condens. Matter, 407, 4837.

[14] Rioux, D.; Vallieres, S.; Besner, S.; Munoz, P.; Mazur, E. and Meunier, M. (2014) Adv. Optical Mater., 2, 176-182.

[15] Link, S. and El-Sayed, M.A. (1999) J. Phys. Chem. B, 103, 8410-8426 and reference therein.

[16] Pinchuk A.O. (2012) J. Phys. Chem. C, 116, 20099 and reference therein.

[17] Raza, S.; Bozhevolnyi, S.I.; Wubs, M. and Mortensen, N.A. (2015) J. Phys.: Condens. Matter, 27, 183204.

[18] Kreibig, U. and Vollmer, M. (1995) Optical Properties of Metal Clusters; Springer, Berlin.

[19] Vial, J.C. and Derrien, J. (1995) Les Editions de Physique and Springer, France.

[20] Rahdar, A.; Arbabi, V. and Ghanbari, H. (2012) Int. J. Chem. Biol. Eng., 6, 1.

[21] Kostic, R. and Stojanovic, D. (2009) Acta. Phys. Pol. A, 116, 4. 